\title{
Calibration of Bi-planar Radiography with a Rangefinder and a Small Calibration Object
}

\author{
Daniel C. Moura ${ }^{1,2}$, Jorge G. Barbosa ${ }^{2}$, João Manuel R. S. Tavares ${ }^{3,4}$, and Ana \\ M. Reis ${ }^{5}$ \\ ${ }^{1}$ Instituto de Eng. Biomédica, Lab. de Sinal e Imagem, Campus da FEUP, Portugal \\ ${ }^{2}$ U. do Porto, Faculdade de Engenharia, Dep. Eng. Informática, Portugal \\ 3 Instituto de Engenharia Mecânica e Gestão Industrial, Campus da FEUP, Portugal \\ ${ }^{4}$ U. do Porto, Faculdade de Engenharia, Dep. de Eng. Mecânica e Gestão Industrial \\ Rua Dr. Roberto Frias, 4200-465 Porto, Portugal \\ \{daniel.moura, jbarbosa, tavares\}@fe.up.pt \\ 5 SMIC, R. Pedro Hispano, 881, 4250-367 Porto, Portugal
}

\begin{abstract}
In this paper we propose a method for geometrical calibration of bi-planar radiography that aims at minimising the impact of calibration objects on the content of radiographs, while remaining affordable. For accomplishing this goal, we propose a small extension to conventional imaging systems: a low-cost rangefinder that enables to estimate some of the geometrical parameters. Even with this extension, a small calibration object is needed for correcting scale.

The proposed method was tested on 17 pairs of radiographs of a phantom object of known dimensions. For calculating scale, only a reference distance of $40 \mathrm{~mm}$ was used. Results show a RMS error of $0.36 \mathrm{~mm}$ with $99 \%$ of the errors inferior to $0.85 \mathrm{~mm}$.

We conclude that the method presented here performs robustly and achieves sub-millimetric accuracy, while remaining affordable and requiring only two radiopaque marks visible in radiographs.
\end{abstract}

\section{Introduction}

Nowadays, Computer Tomography (CT) is the gold standard for 3D reconstructions of bone structures. However, CT scans may not be used for accurate reconstructions of large bone structures, such as the spine, because of the high doses of radiation that are necessary. Additionally, when compared to radiography, CT scans are more expensive, more invasive, less portable, and require patients to be lying down. Therefore, using radiography for obtaining 3D reconstructions and accurate measurements remains an interesting alternative to $\mathrm{CT}$.

Currently, it is possible to do $3 \mathrm{D}$ reconstructions of the spine $[1,2]$, pelvis [3], distal femur [4] and proximal femur [5] with minimal radiation by subjecting the patient just to two radiographs (bi-planar radiography). For achieving this, all these methods require a calibration procedure that must be executed for every examination in order to capture the geometry of the x-ray imaging system. Usually, this calibration is performed using very large calibration apparatus that 
surround the patient and introduce undesirable objects in radiographs. These apparatus are neither practical nor affordable. Not surprisingly, efforts have been made to use smaller calibration objects [6-8], or even to eliminate them at all $[9$, 10]. Currently, and to our knowledge, no method is capable of accurate reconstructions without using calibration objects. Kadoury et al. were able to calculate angular measures from spine radiographs without using any calibration object, but absolute measures scored very poor results and were considered to be unreliable [10]. As for methods that use small calibration objects, reconstruction errors remain considerably higher than when using large apparatus, and a significant number of undesirable objects is still visible in radiographs.

The method proposed in this paper intends to show that it is possible to obtain accurate calibrations of bi-planar radiography using small calibration objects that produce minimal changes to radiographs. For helping accomplishing this goal, we propose a small extension to conventional systems of x-ray imaging: a rangefinder that provides good estimates of some of the geometrical parameters of the system. For accurately assessing this method, experiments were conducted on a phantom object of known geometry.

The remaining of this paper is structured as follow: section 2 describes the proposed method, section 3 presents the validation experiments and its results, and section 4 discusses these results and present the conclusions of this work.

\section{Methods}

This section describes the proposed method. It starts with a general introduction to the problem of radiography calibration and presents a well known but insufficient solution for solving it without calibration objects. Then, an extension to this solution is proposed, which is based on using a rangefinder to determine some of the calibration parameters. Unfortunately, such extension only enables to determine up to scale reconstructions, and therefore we also propose using a small calibration object to correct scale.

\subsection{Radiography calibration}

In bi-planar x-ray systems, the projection of a $3 \mathrm{D}$ point in each of the two radiographs may be calculated as:

$$
\left[\begin{array}{c}
w_{i} \cdot u_{i} \\
w_{i} \cdot v_{i} \\
w_{i}
\end{array}\right]=M_{i} \cdot\left[\begin{array}{c}
X \\
Y \\
Z \\
1
\end{array}\right] \quad \text { for } i=1,2
$$

where for each acquisition $i, M$ is the calibration matrix that describes the projection of the 3D point $(X, Y, Z)$ into image coordinates $(u, v)$ subjected to a scaling factor $w$. For flat x-ray detectors, $M$ may be modelled as:

$$
M_{i}=\left[\begin{array}{cccc}
f_{i} / s & 0 & u_{p_{i}} & 0 \\
0 & f_{i} / s & v_{p_{i}} & 0 \\
0 & 0 & 1 & 0
\end{array}\right] \cdot\left[\begin{array}{cc}
\mathcal{R}_{i} & t_{i} \\
0^{T} & 1
\end{array}\right],
$$


where $f$ is the focal distance (the distance between the $\mathrm{x}$-ray source and the detector $), s$ is the known sampling pitch of the detector, $\left(u_{p}, v_{p}\right)$ is the principal point (2D projection of the x-ray source in the image), and $\mathcal{R}$ and $t$ define the geometrical transformation (represented by a $4 \times 4$ matrix) that aligns the object coordinate system with the source coordinate system. More precisely, $t$ is a translation vector that may be decomposed in $\left(t_{x}, t_{y}, t_{z}\right)$ and $\mathcal{R}$ is a $3 \times 3$ rotation matrix that depends of three angles: an $\alpha$ rotation around the $\mathrm{X}$ axis, a $\beta$ rotation around the $\mathrm{Y}$ axis, and $\gamma$ rotation around the $\mathrm{Z}$ axis.

The goal of the calibration procedure is to find the optimum values of the calibration parameters:

$$
\xi_{i}=\left(f_{i}, u_{p_{i}}, v_{p_{i}}, t_{x_{i}}, t_{y_{i}}, t_{z_{i}}, \alpha_{i}, \beta_{i}, \gamma_{i}\right) \quad \text { for } i=1,2 .
$$

When not using calibration objects, this is usually done by minimising the retroprojection error of a set of point matches marked in the two images $[9,10]$. This problem may be formulated as a least-squares minimisation:

$$
\min _{\xi_{1}^{*}, \xi_{2}^{*}}\left(\sum_{i=1}^{2} \sum_{j=1}^{n}\left\|p_{i j}-\operatorname{prj}\left(\xi_{i}, \operatorname{tri}\left(\xi_{1}, \xi_{2}, p_{1 j}, p_{2 j}\right)\right)\right\|^{2}\right),
$$

where $n$ is the number of marked point matches, $p_{i j}$ is the $j^{\text {th }}$ point marked in image $i, p r j$ is the $2 \mathrm{D}$ projection of a 3D point as defined in equations 1 and $2, t r i$ is a triangulation operation that calculates the $3 \mathrm{D}$ coordinates for a given point match, and $\xi_{1}^{*}, \xi_{2}^{*}$ are the optimised parameters for image 1 and 2 respectively.

Calibration may be accomplished using a standard nonlinear least-squares minimisation algorithm. This class of algorithms needs an initial solution for the calibration parameters, which are then iteratively updated towards reducing the sum of squared distances between the marked and retro-projected points. Unfortunately, the search space of solutions is very large and this procedure gets easily trapped in local minima.

\subsection{Narrowing the search space of solutions}

In order to reduce the search space of solutions, a laser rangefinder was attached to the x-ray machine that allows to estimate the focal length $(f)$ and to have an initial guess of the distance between the object and the x-ray source $\left(t_{z}\right)$. Figure 1 illustrates how the device is attached to the $\mathrm{x}$-ray machine. The device is only capable of measuring the distance between the x-ray machine and the table $\left(d_{m}\right)$. In order to calculate $f$, two more parameters need to be determined: $d_{s}-$ the distance from the $\mathrm{x}$-ray source to the plane of the $\mathrm{x}$-ray device where the $\mathrm{x}$ rays come out, and $d_{d}$-the distance from the table to the x-ray detector. These parameters are fixed for a given imaging system but may be difficult to measure directly. Therefore, we propose finding them indirectly using a radiopaque planar grid (calibration phantom) of known dimensions (figure 3). The procedure described below only needs to be executed once for a given imaging system. 

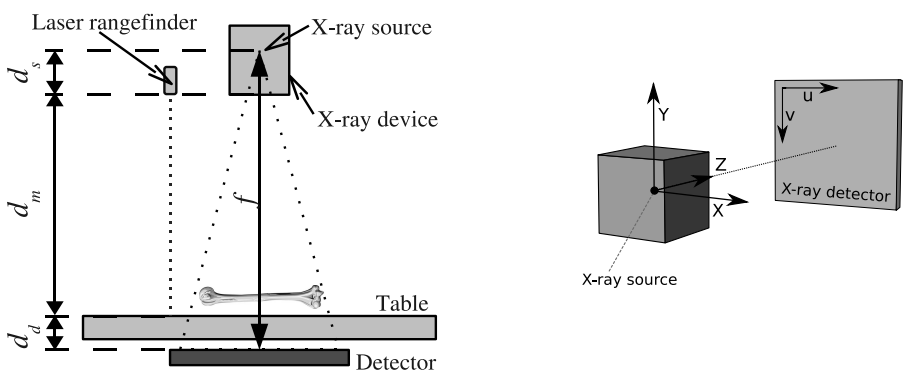

Fig. 1. Illustration of a conventional radiographic imaging system with a laser rangefinder attached (left), and the coordinate system of reference (right).

First, the grid should be placed in the table of the imaging system, roughly centred $\left(t_{x} \simeq 0 \mathrm{~mm}, t_{y} \simeq 0 \mathrm{~mm}\right)$, and roughly parallel to the detector with no tilt $\left(\alpha \simeq 0^{\circ}, \beta \simeq 0^{\circ}, \gamma \simeq 0^{\circ}\right)$. This positioning is easy to accomplish and enables to have a good initial guess for most of the calibration parameters. Parameters $\left(u_{p}, v_{p}\right)$ may be defined as the centre of the image, and parameters $f$ and $t_{z}$ may be redefined in the following way:

$$
\begin{gathered}
f=d_{s}+d_{m}+d_{d}, \\
t_{z}=d_{s}+d_{m}-o_{t} / 2,
\end{gathered}
$$

where $o_{t}$ is the phantom thickness.

However, because the calibration object is planar and because of its positioning, there is an infinite range of solutions for $d_{s}$ and $d_{d}$ that enables to accomplish optimal projections of the grid. This happens because increasing one of these parameters may be compensated by increasing the other. Therefore, several radiographs at different distances $d_{m}$ should be acquired, and, for each of them, the relation between $d_{s}$ and $d_{d}$ should be determined. Then, by crossing results, one may find the point where $d_{s}$ and $d_{d}$ are the same for all setups (figure 2). For finding this relation for a given setup, we ran several optimisations with different values of $d_{s}$ (within an admissible range determined using direct measurements). By fixing $d_{s}$ in each optimisation it is possible to determine the correspondent $d_{d}$ for that setup (figure 2). Therefore, for each setup, and for each tested $d_{s}$, the set of parameters to optimise is:

$$
\zeta=\left(d_{d}, u_{p}, v_{p}, t_{x}, t_{y}, \alpha, \beta, \gamma\right)
$$

Once again, this problem may be formulated as a least-squares minimisation, but, this time, we minimise the projection error of the known $3 \mathrm{D}$ positions of the corners of the phantom squares:

$$
\min _{\zeta^{*}}\left(\sum_{i=1}^{n}\left\|p_{i}-\operatorname{prj}\left(\zeta, d_{m}, d_{s}, o_{t}, P_{i}\right)\right\|^{2}\right),
$$


where $n$ is the number of $2 \mathrm{D}$ points (corners of the phantom) visible on the radiograph, $p_{i}$ is the $i^{\text {th }} 2 \mathrm{D}$ point, $P_{i}$ is the corresponding $3 \mathrm{D}$ coordinate on phantom model, prj is the $2 \mathrm{D}$ projection of a $3 \mathrm{D}$ point as defined in equations 1 and 2 (with the change that $f$ and $t_{z}$ defined in equation 2 should be calculated using equations 5 and 6 respectively), and $\zeta^{*}$ is the optimised set of parameters.

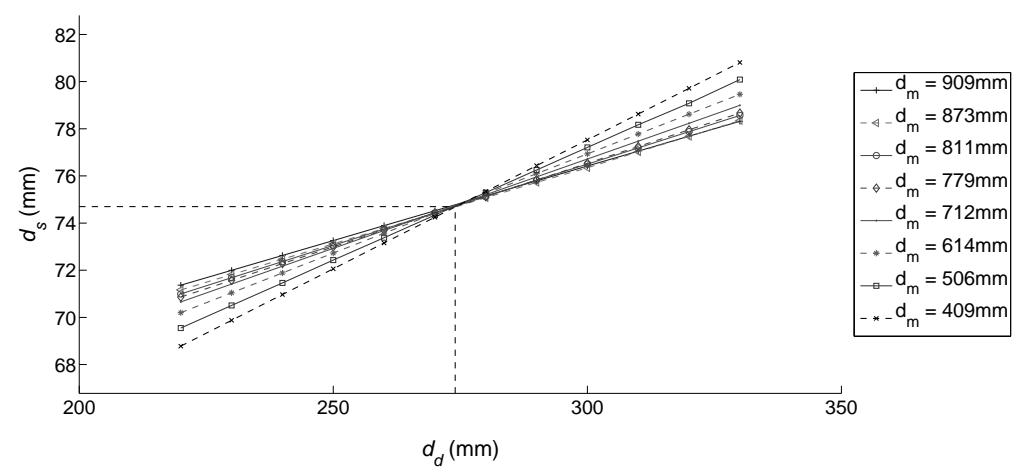

Fig. 2. Variation of $d_{s}$ in function of $d_{d}$ for each setup.

Once $d_{s}$ and $d_{d}$ are calculated for a given system, it is straightforward calculating the focal length and the distance between the x-ray source and the table where the target is positioned just by using a rangefinder. This last value may be used for calculating an initial guess of the target position, since it is usually located near the table.

\subsection{Correcting scale}

In our experiments, using the rangefinder only enabled determining up to scale solutions for the 3D coordinates. For correcting scale, a reference measure is needed. Such measure may be obtained using a small calibration object composed by only two radiopaque parts placed at a known distance, which should be attached to the patient and be visible in both radiographs. The scaling factor may be calculated as the ratio between the real distance between the two radiopaque objects and the distance between the reconstructed $3 \mathrm{D}$ coordinates of the same objects.

\section{$3 \quad$ Results}

\subsection{Determining $d_{s}$ and $d_{d}$}

For determining parameters $d_{s}$ and $d_{d}$ we built a phantom made of stainless steel (AISI 304) with dimensions of $380 \times 380 \times 1 \mathrm{~mm}$ and laser cut squares of $20.0 \pm$ 
$0.2 \mathrm{~mm}$ (figure 3). Eight radiographs of the phantom were acquired at different distances $\left(d_{m}\right.$ ranging $\left.409-909 \mathrm{~mm}\right)$ while keeping all other parameters constant. For each radiography the distance between the x-ray device and the table $\left(d_{m}\right)$ was measured using a laser rangefinder (typical error of $\pm 1.5 \mathrm{~mm}$, maximum error of $\pm 3.0 \mathrm{~mm}$, and range of operation of $0.05-50 \mathrm{~m})$.

For the optimisation process, we used the coordinates of the corners of the phantom. For each image the 2D coordinates of the corners where extracted semi-automatically with the Camera Calibration Toolbox for Matlab ${ }^{6}$ and then optimised with OpenCV ${ }^{7}$.

Figure 2 shows the relation between $d_{s}$ and $d_{d}$ for each setup. We choose the value of $d_{s}$ where the standard deviation of $d_{d}$ was minimum (which was $0.03 \mathrm{~mm}$ ). Parameter $d_{d}$ was calculated by averaging the values of $d_{d}$ of all setups for the selected $d_{s}$.

\subsection{Assessment of the calibration method}

For evaluating the proposed calibration method, we used the same phantom from the previous procedure. This time, eight radiographs were taken with the phantom at different positions and with different orientations. Distance $d_{m}$ was the same for all setups $\left(d_{m}=909 \mathrm{~mm}\right)$, resulting in a focal length of $f=1257.7 \mathrm{~mm}$ and in a distance between the x-ray source and the table of $1183.0 \mathrm{~mm}$. Film size was of $14^{\prime \prime} \times 17^{\prime \prime}(355.6 \times 431.8 \mathrm{~mm})$, scanned with a sampling pitch of 175.0 $\mu \mathrm{m} /$ pixel, resulting in images with a resolution of $2010 \times 2446$ pixels.

The eight radiographs were combined in a total of 17 pairs (out of 28 possible combinations). Only pairs of radiographs with considerably different pose were considered. Pairs of radiographs with near pose were discarded because are less tolerable to triangulation errors (when pose is similar triangulation lines tend to intersect at infinity because they are close to parallel).

As stated previously, the minimisation algorithm requires an initial estimate of the calibration parameters. Parameters $\left(u_{p}, v_{p}\right)$ were initialised with the $2 \mathrm{D}$ coordinates of the image centre, and $t$ and $\mathcal{R}$ where roughly estimated in the following way:

$-t_{x}$ and $t_{y}$ were always initialised with zero (we assumed that the object was roughly centred in the radiograph, even if it was not);

$-t_{z}$ was initialised with $d_{s}+d_{m}$ when its centre was near the table; when the centre was farther away from the table due to its pose, half of the object width was subtracted;

$-\alpha, \beta$, and $\gamma$ were roughly provided in a $10^{\circ}$ resolution scale.

Figure 3 shows three examples of the initial guesses for three radiographs, and table 1 summarises the errors for all of them. These errors were calculated by comparing the initial guess for each radiograph with the parameters that achieved optimal solutions (minimum projection error) when projecting a 3D model of the phantom on the correspondent radiograph.

\footnotetext{
${ }^{6}$ http://www.vision.caltech.edu/bouguetj/calib_doc/ (accessed Dec-2007)

${ }^{7}$ http://www.intel.com/technology/computing/opencv/ (accessed Dec-2007)
} 


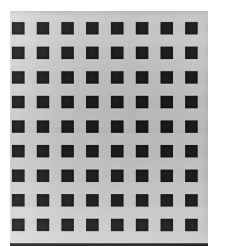

$(0,0,1183)$

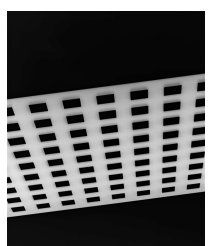

$(0,0,993)$

$\left(60^{\circ}, 0^{\circ}, 0^{\circ}\right)$

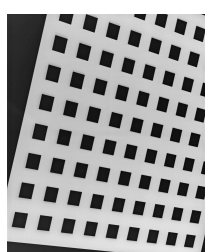

$(0,0,993)$

$\left(0^{\circ},-40^{\circ}, 10^{\circ}\right)$

Fig. 3. Example of three radiographs of the phantom and the initial guess for $\left(t_{x}, t_{y}, t_{z}\right)$ in $\mathrm{mm}$ and $(\alpha, \beta, \gamma)$ in degrees.

Table 1. Absolute errors for position $\left(t_{x}, t_{y}, t_{z}\right)$ and orientation $(\alpha, \beta, \gamma)$ of the initial guess for each radiograph.

\begin{tabular}{lrrrrrr}
\hline & $\begin{array}{r}t_{x} \\
(\mathrm{~mm})\end{array}$ & $\begin{array}{r}t_{y} \\
(\mathrm{~mm})\end{array}$ & $\begin{array}{r}t_{z} \\
(\mathrm{~mm})\end{array}$ & $\begin{array}{r}\alpha \\
(\mathrm{deg})\end{array}$ & $\begin{array}{r}\beta \\
(\mathrm{deg})\end{array}$ & $\begin{array}{r}\gamma \\
(\mathrm{deg})\end{array}$ \\
\hline Mean & 17.7 & 21.0 & 21.9 & 0.9 & 1.7 & 2.4 \\
SD & 7.2 & 13.4 & 21.6 & 1.6 & 1.6 & 2.5 \\
Max & 30.5 & 43.2 & 58.6 & 3.8 & 3.9 & 6.9 \\
\hline
\end{tabular}

As stated in the previous section, the calibration process tries to minimise the retro-projection errors of a set of point matches marked in a pair of images. We used the corners of the squares of the phantom that were visible in both images, which in average originated 199 point matches. These points were semiautomatically extracted using the previously described procedure. The full set of point matches was used in the first two experiments for evaluating the method with maximal input. In addition, a third experiment was conducted to evaluate the effect of using smaller sets of points.

The optimisation algorithm that was used was the Matlab large-scale algorithm for nonlinear least-squares problems, which shown a superior performance than the conventional approach of using the Levenberg-Marquardt algorithm (e.g. some of the works that use this last approach: [7-10]).

For simulating the calibration object that should be utilised for determining the scaling factor, reference distances of $40 \mathrm{~mm}$ were used, which correspond to two consecutive squares of the phantom. For the first experiment we tested scaling with 50 reference distances uniformly distributed by the part of the phantom that was visible in both radiographs. First, the $3 \mathrm{D}$ coordinates of every point match were calculated using the optimised parameters. Then, the $3 \mathrm{D}$ points were scaled (using one reference distance) and aligned with a 3D model of the phantom. Finally, 3D errors were computed as the Euclidean distances between the calculated points and the corresponding points in the model. Figure 4(a) shows an histogram of the errors for the complete experiment. RMS error was $0.36 \mathrm{~mm}$ and $99 \%$ of the errors were inferior to $0.85 \mathrm{~mm}$. 
A second experiment was carried out for testing the proposed procedure in less optimal conditions of point matches identification. This was done by adding uniformly distributed noise to the $2 \mathrm{D}$ coordinates of the phantom's corners that were previously extracted using the Camera Calibration Toolbox for Matlab and OpenCV. Then, the previous experiment was repeated (17 pairs of images $\times 50$ reference distances) starting with no noise, and adding up to \pm 15 pixels to each coordinate of every point. We decided not adding noise to the two points that defined the reference distance because in real cases these points would be represented by two radiopaque objects that would be easy to identify accurately. Results are presented in figure 4(b) showing the relation between pixel noise and mean $3 \mathrm{D}$ error.

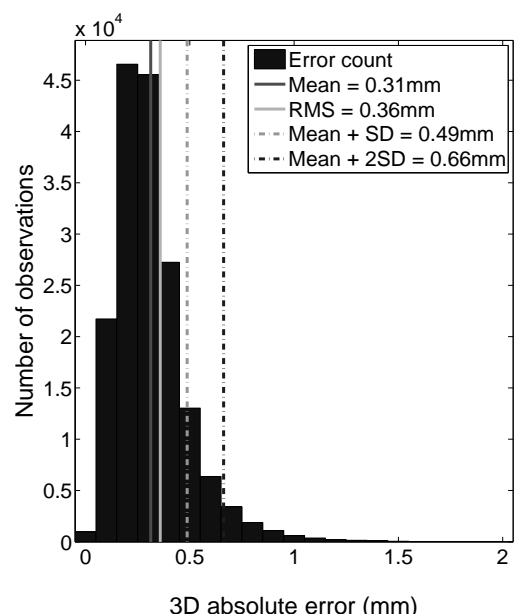

(a)

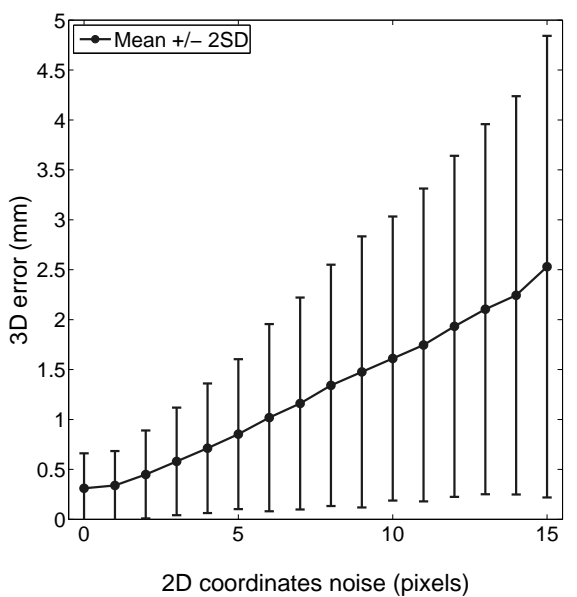

(b)

Fig. 4. Histogram of the 3D errors of the phantom reconstruction (a), and 3D errors vs. noise in landmarks location (b).

A final experiment was made to determine if the method was capable of achieving good results with smaller sets of points. We tested using sets of 67,33 and 23 average point matches, which are in the range of values that are typically used in this kind of procedures. For each set, the first experiment was repeated with no noise added to point matches, and then with uniformly distributed noise of \pm 5 and \pm 10 pixels. Table 2 shows the results for this experiment.

\section{Conclusions}

This paper presents a method for bi-planar radiography calibration that uses a low-cost rangefinder for narrowing the search space of solutions. This enhancement enables to improve the calibration performance of conventional x-ray 
Table 2. 3D reconstruction error vs. number of point matches and noise in point matches location.

\begin{tabular}{cccc}
\hline \multirow{2}{*}{ Number of matches } & \multicolumn{3}{c}{$3 \mathrm{D}$ RMS reconstruction errors $(\mathrm{mm})$} \\
\cline { 2 - 4 } & No noise & \pm 5 pixels noise & \pm 10 pixels noise \\
\hline 199 & 0.36 & 0.93 & 1.76 \\
67 & 0.36 & 0.98 & 1.80 \\
33 & 0.38 & 0.98 & 1.86 \\
23 & 0.39 & 1.21 & 2.10 \\
\hline
\end{tabular}

imaging systems without affecting radiographs and with minor inconvenience for patients and technicians. Results show that this method is robust and offers submillimetric accuracy even when the initial guess of the calibration parameters is rather rough. Results also show that the quality of the calibration depends of the quality of point matches identification in radiographs. However, this dependence is close to linear for the tested range of noise, and when uniformly distributed noise of \pm 5 pixels is added to each coordinate of every point, the RMS error remains inferior to $1 \mathrm{~mm}$. This demonstrates the robustness of the proposed method since it achieves acceptable errors even when using a pessimist distribution of noise where all values are equiprobable. Additionally, when decreasing the average number of point matches to 33 the method shows a very small increase of the error, enabling to achieve almost the same results with much less input. This number of point matches is inside the range of typical values used in other calibration procedures of this kind.

For achieving these results a small calibration object is needed (we used a $40 \mathrm{~mm}$ reference distance in our experiments). Its only role is determining the scaling factor that should be applied for obtaining real-world units. Thus, this object may be discarded if the goal is only to obtain shape, or angular and relative measurements. When compared to other works [6-8], the proposed calibration object has less impact in the content of radiographs (only two radioopaque marks are needed). Results may not yet be compared because the other studies use anatomical structures, either in vivo or in vitro, and therefore, score higher errors.

A possible disadvantage of this technique is that it requires an estimation of the rotation and translation parameters. This may not be a problem for some kind of examinations, such as spine radiography, where frontal and lateral radiographs are usually acquired. In these cases an initial estimation is very simple to obtain because the patient is typically with $0^{\circ}$ rotation for all axis in the first radiograph and then, for the second radiograph, he/she only experiences a $90^{\circ}$ rotation around one of the axis. Either way, for best results the two acquisitions should not be taken with near poses for preventing triangulation errors, which is the case of spine radiographs. 
Ongoing experiments with simulation of spine radiography using in vivo data show that the length of the calibration object should be higher $(10-12 \mathrm{~cm})$ but reconstruction errors remain lower that the published for in vivo and in vitro studies.

\section{Acknowledgements}

The first author thanks Fundação para a Ciência e a Tecnologia for his PhD scholarship (SFRH/BD/31449/2006). The authors would also like to express their gratitude to Instituto de Neurociências and to SMIC.

\section{References}

1. Mitulescu, A., Skalli, W., Mitton, D., De Guise, J.: Three-dimensional surface rendering reconstruction of scoliotic vertebrae using a non stereo-corresponding points technique. European Spine Journal 11 (2002) 344-352

2. Pomero, V., Mitton, D., Laporte, S., de Guise, J.A., Skalli, W.: Fast accurate stereoradiographic 3d-reconstruction of the spine using a combined geometric and statistic model. Clinical Biomechanics 19 (2004) 240-247

3. Mitton, D., Deschênes, S., Laporte, S., Godbout, B., Bertrand, S., de Guise, J.A., Skalli, W.: 3d reconstruction of the pelvis from bi-planar radiography. Computer Methods in Biomechanics \& Biomedical Engineering 9 (2006) 1-5

4. Laporte, S., Skalli, W., de Guise, J., Lavaste, F., Mitton, D.: A biplanar reconstruction method based on $2 \mathrm{~d}$ and $3 \mathrm{~d}$ contours: Application to the distal femur. Computer Methods in Biomechanics \& Biomedical Engineering 6 (2003) 1-6

5. Baudoin, A., Skalli, W., Mitton, D.: Parametric subject-specific model for in vivo $3 \mathrm{~d}$ reconstruction using bi-planar x-rays: Application to the upper femoral extremity. Comput.-Assisted Radiol. Surg. 2 (2007) S112-S114

6. Cheriet, F., Delorme, S., Dansereau, J., Aubin, C., De Guise, J., Labelle, H.: Intraoperative $3 \mathrm{~d}$ reconstruction of the scoliotic spine from radiographs. Annales de Chirurgie 53 (1999) 808-815

7. Kadoury, S., Cheriet, F., Laporte, C., Labelle, H.: A versatile 3d reconstruction system of the spine and pelvis for clinical assessment of spinal deformities. Medical \& Biological Engineering \& Computing 45 (2007) 591-602

8. Cheriet, F., Laporte, C., Kadoury, S., Labelle, H., Dansereau, J.: A novel system for the 3 -d reconstruction of the human spine and rib cage from biplanar x-ray images. IEEE Transactions on Biomedical Engineering 54 (2007) 1356-1358

9. Cheriet, F., Dansereau, J., Petit, Y., Aubin, C., Labelle, H., De Guise, J.A.: Towards the self-calibration of a multiview radiographic imaging system for the $3 \mathrm{~d}$ reconstruction of the human spine and rib cage. International Journal of Pattern Recognition \& Artificial Intelligence 13 (1999) 761-779

10. Kadoury, S., Cheriet, F., Dansereau, J., Labelle, H.: Three-dimensional reconstruction of the scoliotic spine and pelvis from uncalibrated biplanar x-ray images. Journal of Spinal Disorders \& Techniques 20 (2007) 160-167 